\title{
Optimal Synthesis of Horizontally Aligned Single-Walled Carbon Nanotubes and Their Biofunctionalization for Biosensing Applications
}

\author{
Dawoon Jung, ${ }^{1}$ Hyun Woo Park, ${ }^{1}$ Gidong Ma, ${ }^{1}$ Chang Young Lee, ${ }^{2}$ \\ Taeyun Kwon, ${ }^{3}$ and Jae-Hee Han ${ }^{1}$ \\ ${ }^{1}$ Department of Energy IT, Gachon University, Seongnam 13120, Republic of Korea \\ ${ }^{2}$ School of Energy and Chemical Engineering, Ulsan National Institute of Science and Technology (UNIST), \\ Ulsan 44919, Republic of Korea \\ ${ }^{3}$ SKKU Advanced Institute of Nano Technology, Sungkyunkwan University (SKKU), Suwon 16419, Republic of Korea
}

Correspondence should be addressed to Taeyun Kwon; taeyunkwon@gmail.com and Jae-Hee Han; jhhan388@gachon.ac.kr

Received 17 February 2016; Accepted 30 May 2016

Academic Editor: Serdal Kirmizialtin

Copyright (C) 2016 Dawoon Jung et al. This is an open access article distributed under the Creative Commons Attribution License, which permits unrestricted use, distribution, and reproduction in any medium, provided the original work is properly cited.

\begin{abstract}
As an influential candidate for highly sensitive biomolecule sensor, which can capture disease related biomolecules, carbon nanotube is useful material due to its unique properties. To adopt as a sensing platform, it is strongly needed to find optimal refined synthetic condition. In order to find the optimal synthetic conditions of horizontally aligned CNT, we performed quantity control of the mixed gases of $\mathrm{H}_{2}$ and $\mathrm{CH}_{4}$ injected. We successfully find that the formation of amorphous-like carbon was critically affected by some gas condition such as the flow rate of injected gases and ratios of gas mixture. Moreover, it should be noted that our horizontally aligned carbon nanotube array platform developed would offer another potential in developing nanoscale light source, where light emission results from electron-hole carrier recombination.
\end{abstract}

\section{Introduction}

In the past decades, it has been attractive to the community to develop the novel bioassay toolkits, which allow the fast, reliable, label-free, and highly sensitive detection of specific biological species, eventually resulting in early diagnosis of diseases such as cancer [1-4]. To the best of our knowledge, the detection sensitivity of conventional toolkits, such as DNA microarray, based on fluorescence imaging is not as good as that required for early cancer diagnosis due to the relatively large sensing surface and ineffectiveness of fluorescence, for example, low durability, and fluorescence quenching [5-7].

Scanning probe microscopy- (SPM-) based detection methods have recently served as a sensing toolkit that is able to sensitively identify the specific target biomolecules owing to the high spatial resolution of AFM (being even up to nanometer scale) [8-13]. For instance, tapping mode Atomic Force Microscopy (tmAFM) and Kelvin probe force microscopy (KPFM) have enabled the sensitive detection of biomolecules such as DNA, RNA, and proteins $[8,13]$. In addition, SPM does not require the cumbersome processing of samples such as the labeling of fluorescence dyes to target biomolecules, which implies that the fabrication of the sensing substrate is cost-effective and straightforward.

In addition to using SPM as an imaging toolkit, the preparation of nanoscale pattern is necessary in order for SPM-based sensing to achieve the high detection sensitivity. One of ways to prepare the nanoscale pattern is to consider carbon nanotube (CNT), which is useful material due to its unique properties. Specifically, it is easy to chemically modify the surface of CNT, particularly chemically attaching biological probes (e.g., aptamer, DNA, and RNA) to CNT surface. This easiness of the biological functionalization of CNT surface leads to the preparation of carbon nanotubepatterned surface that is able to specifically and selectively capture target biomolecules. In addition, as the diameter of 
CNT is in the order of 1 to at most $10 \mathrm{~nm}$, the very minute amount of target biomolecules can be specifically captured to the small area of CNT-patterned surface, which indicates the high detection sensitivity of CNT-patterned surface.

Based on aforementioned CNT-patterned surface along with SPM imaging technique, we have shown that a horizontally aligned carbon nanotube- (HACNT-) patterned surface enables the specific detection of biomolecules with ultrasensitivity for early cancer diagnostics $[14,15]$. In particular, it is shown that HACNT-patterned surface-based detection system is able to sense specific target biomolecules such as proteins (e.g., thrombin) and tumor marker. The detection limit of our detection system based on SPM imaging and HACNT-patterned surface is as low as single-molecule level, which is much better than the detection limit of other conventional bioassay toolkits.

For sensitive detection based on aforementioned CNTpatterned surface, it is important to prepare the optimally patterned CNTs on the surface. In our previous works, the optimal patterning of CNTs on the surface has not been fully studied yet. For instance, when CNT pattern is not optimally designed, it is likely that amorphous carbon can undesirably be formed when CNT is chemically grown; this amorphous carbon may prevent the CNT-patterned surface from exhibiting the high detection sensitivity. In this work, we provide the optimal fabrication condition that allows us to prepare the well-designed CNT-patterned surface, which can serve as a biosensing platform. Moreover, we describe the optimal condition for chemical functionalization of CNTs patterned on the surface for specific and selective detection. In particular, we delineate the functionalization of CNTs with rhodamine dyes. The surface patterned with CNTs functionalized with rhodamine can act as a nanoscale optical device that may respond to the environmental change $[16,17]$.

\section{Experimental Methods}

2.1. Carbon Nanotube Synthesis and Functionalization. In order to synthesize the HACNT array, a thermal chemical vapor deposition (CVD) system (SciEnTech Co., Ltd.) was employed. In our previous work, the mechanism and details of thermal CVD system to grow the array are well described $[14,15,18]$. In brief, the thermal CVD system we used in this work consists of hydrocarbon feedstock gas (methane, $\mathrm{CH}_{4}$, 99.9\%) as atomic carbon source for the nanotube growth, both hydrogen $\left(\mathrm{H}_{2}, 99.999 \%\right)$ and argon ( $\left.\mathrm{Ar}, 99.999 \%\right)$ as catalytic carrier gases, and a $\mathrm{SiO}_{2} / \mathrm{Si}$ substrate with iron (Fe) catalyst layer. The growth time was fixed to 3 hours; the growth temperature varied between 1000 and $1100^{\circ} \mathrm{C}$ at atmospheric pressure. To functionalize the synthesized HACNT array with 4-aminophenyl-diazonium tetrafluoroborate salt $\left(\mathrm{NH}_{2}\right.$-diazonium), the array was dipped into a solution of $\mathrm{NH}_{2}$-diazonium with a small amount of $\mathrm{NaOH}$ for catalyst for this chemical reaction with stirring at $50^{\circ} \mathrm{C}$ for 1 hour. After the $\mathrm{NH}_{2}$-diazonium functionalization, the array was redipped into a solution of rhodamine-b-isothiocyanate (RITC) dyes with stirring at room temperature for 10 hours. The morphology of the HACNT array after synthesis and a subsequent functionalization was characterized using scanning electron microscopy (SEM, Hitachi S-4700).

2.2. Atomic Force Microscopy Imaging. All images of CNT were acquired using a tapping mode at the scanned location. The SPM-based measurement was performed using Innova (Veeco Inc., Santa Barbara, CA, USA) with Nanocontroller V (Veeco Inc.) under an ambient condition at room temperature. To obtain images, we have utilized TESP-V2 tip (Veeco Inc.) as a scanning probe. Here, TESP-V2 tip has the normal resonant frequency of $320 \mathrm{kHz}$ and tip radius of $\sim 8 \mathrm{~nm}$. The tapping mode AFM (tmAFM) images were acquired based on scanning speed of $1 \mu \mathrm{m} / \mathrm{s}$, which is suitable to guarantee the high-quality images. We obtained the tmAFM images for the scan size of $2 \mu \mathrm{m} \times 2 \mu \mathrm{m}$, which is a proper scan size to image a couple of CNTs on the substrate. All SPM images were generated from Nanoscope software V7.2.

2.3. Raman Spectroscopy. The degree of the functionalization of the HACNT array with $\mathrm{NH}_{2}$-diazonium and RITC was characterized using Raman spectroscopy (WITec, Alpha 300 $\mathrm{M}+, 532 \mathrm{~nm}$ laser excitation, and $300 \mathrm{~nm}$ spatial resolution). In particular, Raman spectra of the sample were obtained after the sample was fully dried at room temperature. Raman mapping was carried out with a spectral area of $10 \times 10 \mu \mathrm{m}^{2}$ using a customized built-in software, where points per line and scan speed were 100 and 13 seconds/line, respectively.

\section{Results and Discussion}

Figure 1(a) shows a schematic illustration of a horizontally aligned carbon nanotube (HACNT) array on a $\mathrm{SiO}_{2} / \mathrm{Si}$ substrate grown by chemical vapor deposition (CVD) method. In the case of HACNT array growth, individual carbon nanotubes are generally known to get nucleated from catalyst metals prepatterned at the one end of the substrate and then subsequently extend their length with a process time through the kite mechanism by which carbon nanotubes being grown are floating above the underlying substrate in the same direction with feedstock gas flow injected into a reactor during synthesis [19]. Figure 1(b) depicts a schematic diagram representing a process of surface functionalization of individual carbon nanotubes of the HACNT array with $\mathrm{NH}_{2}$ diazonium after the array synthesis and then subsequent RITC functionalization. The amine groups functionalized on the outer surface of carbon nanotubes are generally expected to act as an electron donor to give electrons towards the nanotube because of their relatively high reduction potential over the valence band of the semiconducting single-walled carbon nanotubes $[20,21]$. In addition, the $\mathrm{NH}_{2}$-diazonium functionalization on the nanotubes would allow mediation of a further functionalization of RITC on the $\mathrm{NH}_{2}$-diazonium functionalized nanotube assembly by replacing $\mathrm{NH}_{2}$-group with RITC, which exhibits fluorescence in the visible light wavelength regime [22].

Scanning electron microscopic (SEM) images of the HACNT arrays are shown in Figure 2, where the effect of total flow rate of feedstock gases during the synthesis on the 


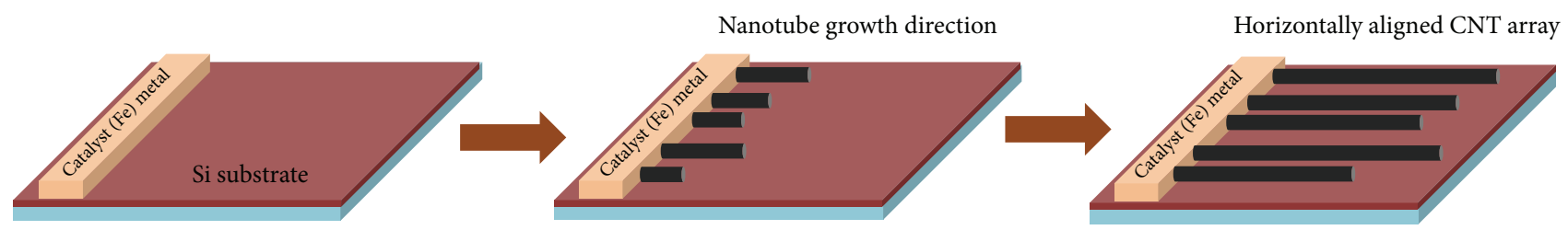

(a)

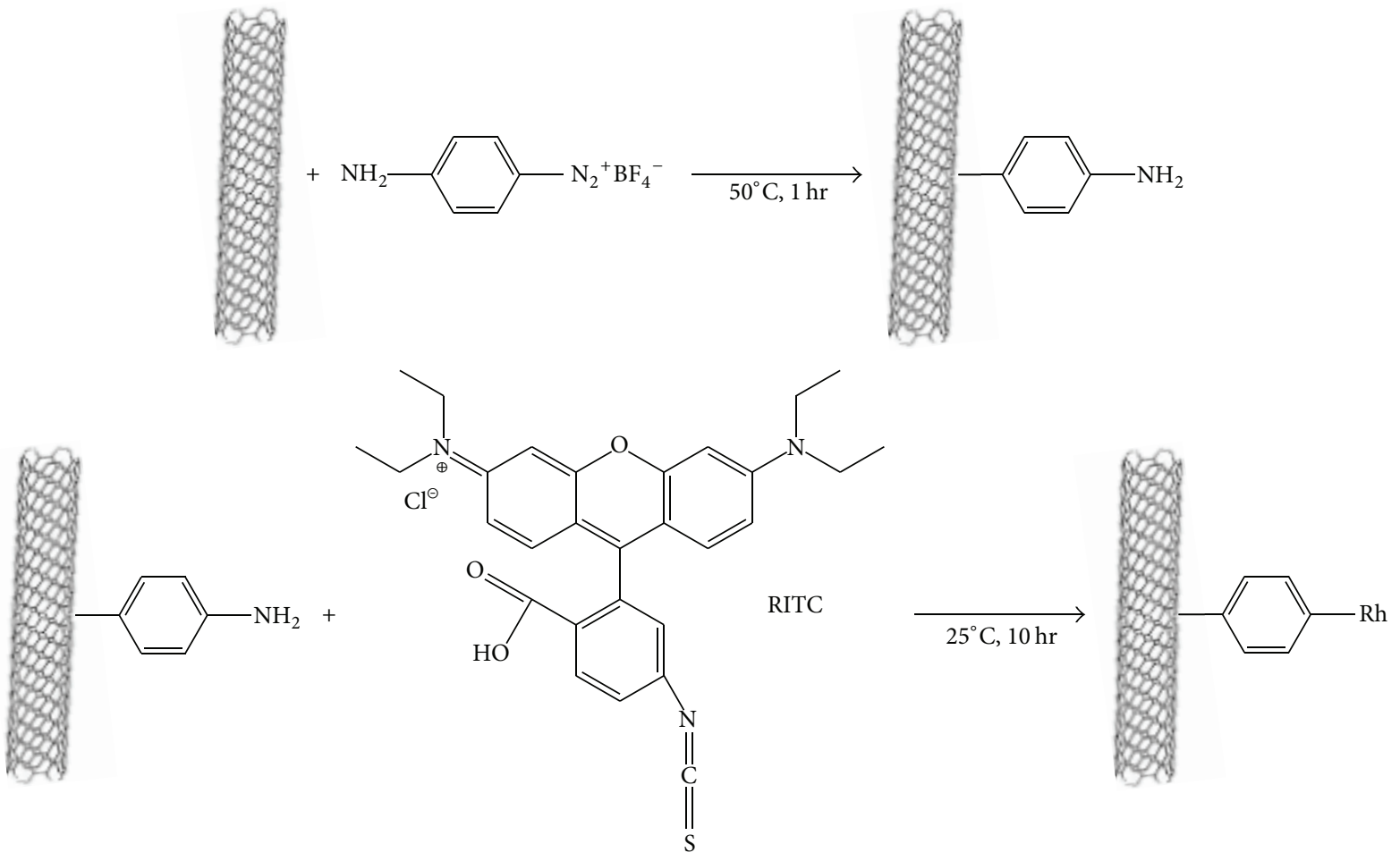

(b)

FIGURE 1: Schematic illustrations of (a) fabrication of a horizontally aligned carbon nanotube (HACNT) array by chemical vapor deposition (CVD) method on a Si substrate and (b) subsequent functionalization of HACNT with $\mathrm{NH}_{2}$-diazonium, followed by rhodamine dye moieties.

growth morphology and density of the arrays is provided when a relative flow rate ratio of hydrogen $\left(\mathrm{H}_{2}\right)$ and methane $\left(\mathrm{CH}_{4}\right)$ gas was kept constant as two. In each subset of the Figure 2, the left (or right) image shows the appearance of the nanotubes in the array grown near (or far away from) the Fe catalyst metal in the nanotube growth region, which is indicated by a red arrow in an upper-left panel of the Figure 2. As total flow rate of the feedstock gases was increased from 4.5 to 9.0 stand cubic centimeter (SCCM), the growth density of each sample was found to be different although the morphologies seemed to be similar (Figures 2(a)-2(d)). Interestingly, when the total flow rate was 9 SCCM (Figure 2(d)), it was hard to find the initial nucleation of the nanotubes allowing their substantial growth afterwards. This result would be possibly because the hydrogen atoms could either etch away the carbon atoms, which are thermally dissociated from $\mathrm{CH}_{4}$ feedstock gas, or replace the dangling bonds of the carbon atoms prior to forming a normal nanotube structure [23]. It is expected that there would be a certain total flow rate that corresponds to the optimal quantity making the nanotubes initiate their growth even if a relative flow rate ratio of the feedstock gases was maintained to be constant. Otherwise, the growth of the HACNT array would be deactivated (Figure 2(d)).

To determine the effect of the relative flow rate ratio of $\mathrm{H}_{2}$ and $\mathrm{CH}_{4}$ compared to the result shown in Figure 2, where the flow ratio is two, the flow ratio using the same gases was slightly decreased to one and a half (Figure 3 ). Then, as similarly shown in Figure 2, we have systematically increased the total flow rate of the feedstock gases from 3.75 to 7.5 SCCM (Figures 3(a)-3(c)). In this case, the flow rate of $\mathrm{H}_{2}$ gas injected was reduced by $75 \%$ at a given flow rate of $\mathrm{CH}_{4}$ gas as compared with that in Figure 2. As the total flow rate was increased (Figures 3(a)-3(c)), the morphology and density of the HACNT arrays were markedly changed as compared with the result shown in Figure 2. More specifically, when the total flow rate was increased from 3.75 SCCM (Figure 3(a)) to 6.25 SCCM (Figure 3(b)) by a factor of $\sim 1.7$, it was observed that the surface of the carbon nanotubes in the array grown was covered by a large amount of the amorphous-like carbon. This morphological appearance is quite obviously compared with that shown in Figure 2, 
$\longrightarrow$ Nanotube growth direction for all of the samples

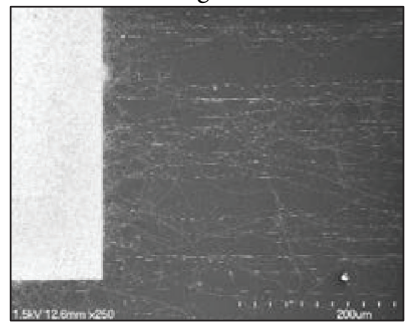

(a)
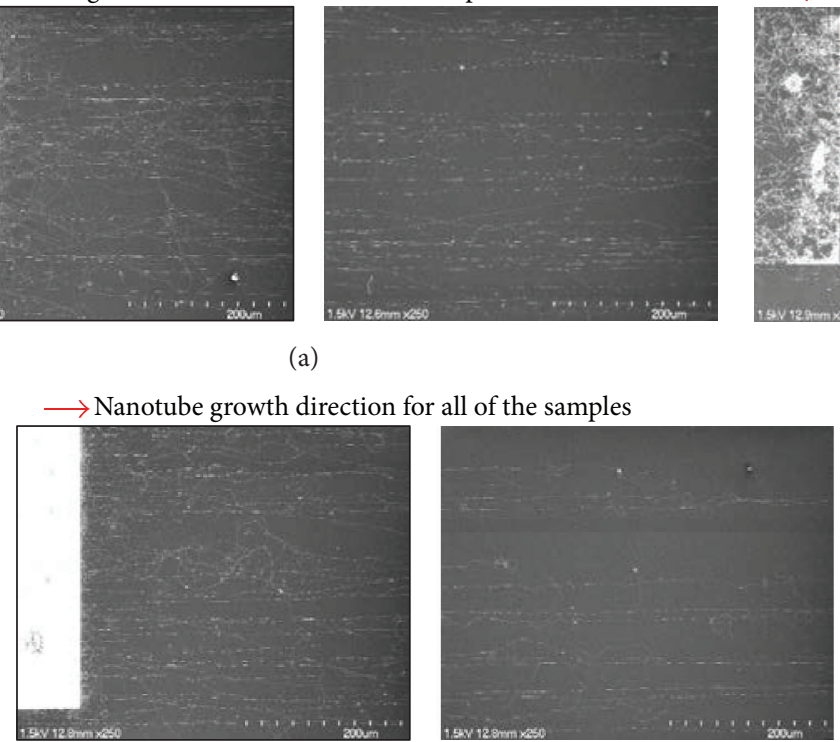

(c) $\longrightarrow$ Nanotube growth direction for all of the samples
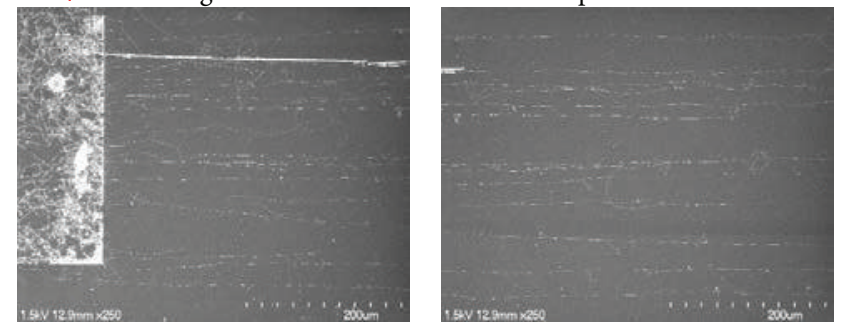

(b)

$\longrightarrow$ Nanotube growth direction for all of the samples

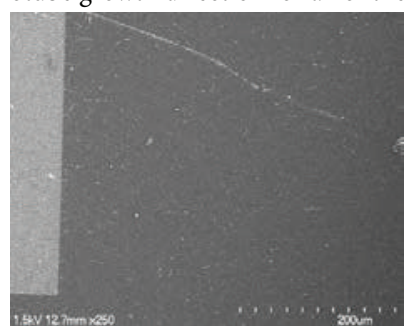

(d)

FIGURE 2: Scanning electron microscopy (SEM) images representing an effect of total flow rate (in unit of standard cubic centimeter (SCCM) of feedstock gases while maintaining a relative flow rate ratio of $\mathrm{H}_{2}$ and $\mathrm{CH}_{4}$, which is two, during HACNT arrays growth on their morphologies and densities. Total flow rate varies at (a) 4.5, (b) 6.0, (c) 7.5, and (d) 9.0, respectively. Note that no tubes were nearly grown at the total flow rate of 9.0. Left (or right) image of each subfigure set corresponds to a regime of the array grown near (or far from) Fe catalyst in the growth direction.

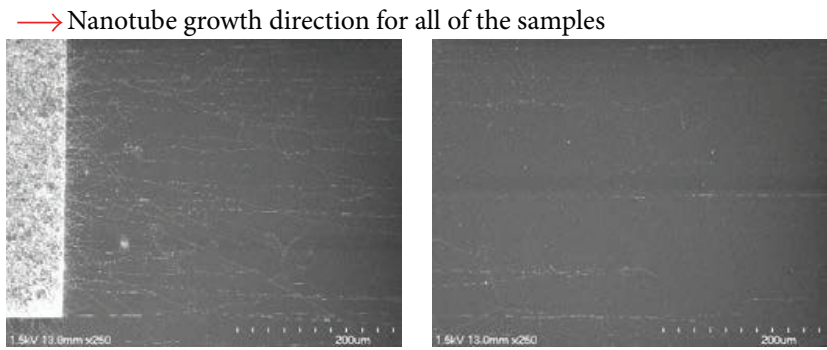

(a) $\longrightarrow$ Nanotube growth direction for all of the samples
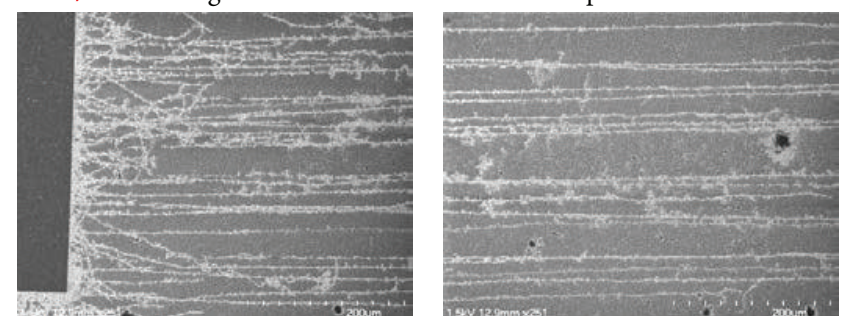

(b)

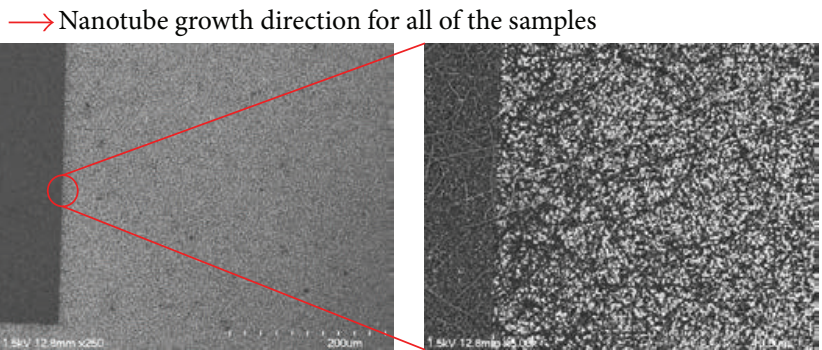

(c)

FIGURE 3: SEM images indicating an effect of a different relative flow rate of $\mathrm{H}_{2}$ and $\mathrm{CH}_{4}$ gases, which is one and a half, compared to the results of Figure 2, on the morphologies and densities of HACNT arrays grown with the total flow rates of (a) 3.75, (b) 6.25, and (c) 7.5, respectively. Note that few tubes were grown and covered with amorphous-like carbon debris at the total flow rate of 7.5.

where the same amount of the total flow rate was increased (Figures 2(a) and 2(c)). Moreover, when the total flow rate was increased to 7.5 SCCM, which equals an increase by a factor of 2 compared to that shown in Figure 3(a), the HACNT array was found to be rarely grown, and most of the substrate seemed to be coated with thick layers of the amorphous-like carbon. In brief, with the results of Figures 2 and 3 mentioned above, the mechanism of patterned carbon nanotube growth can be described as follows: if the relative flow rate ratio of $\mathrm{H}_{2}$ and $\mathrm{CH}_{4}$ is decreased by a certain percentage (despite the 


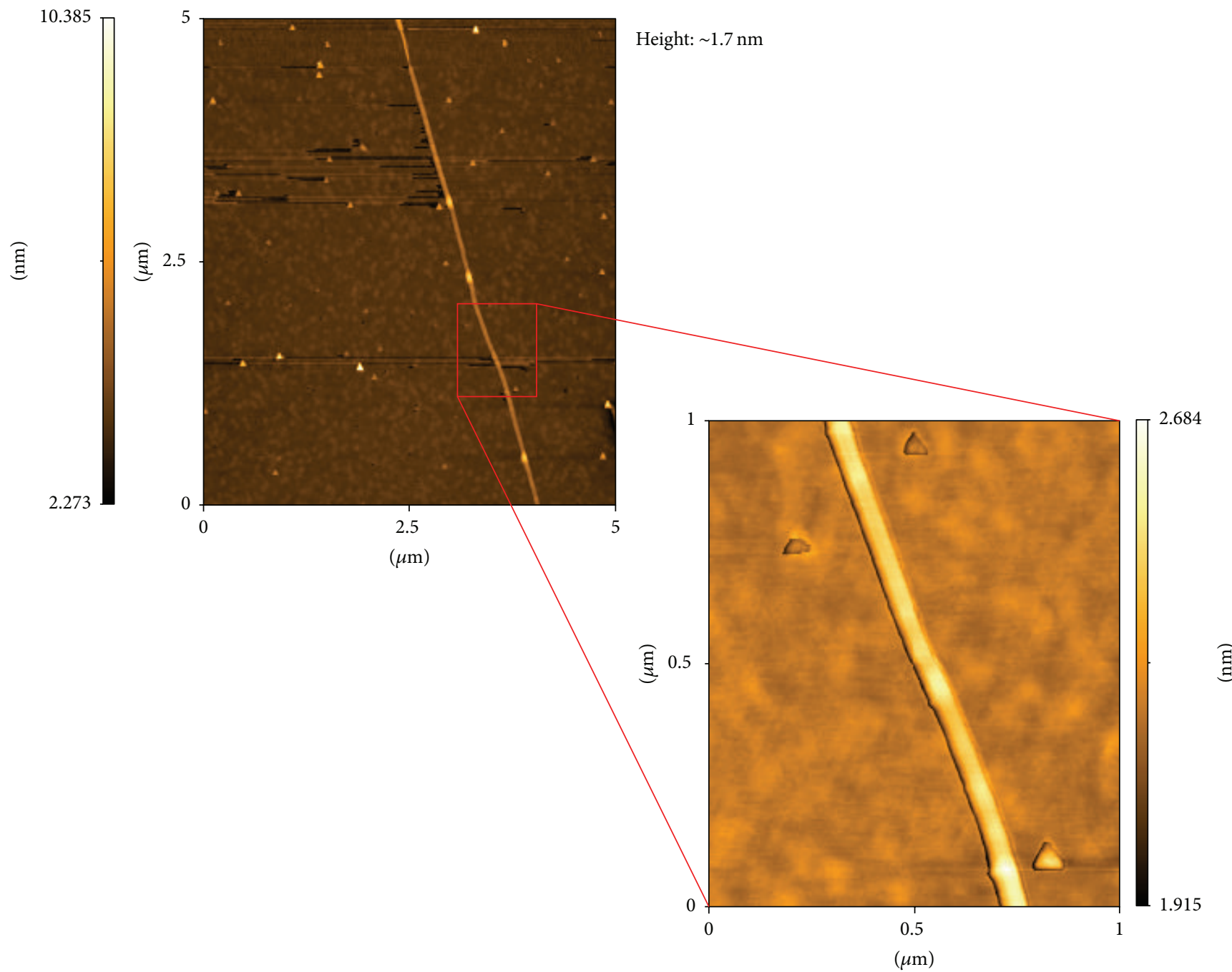

FIgURE 4: Atomic force microscopy (AFM) images of a representative HACNT array.

total flow rates of feedstock gases injected into a reactor being the same), the carbon atoms produced from $\mathrm{CH}_{4}$ gas would not be able to properly form a lattice structure of the carbon nanotube due to decrease in numbers of the hydrogen atoms created from $\mathrm{H}_{2}$ gas, which are supposed to act as scavengers for the carbon atoms in the gas phase reaction [24].

Figure 4 shows AFM images of the HACNT-patterned surface. As shown in Figure 4, the diameter of CNT horizontally grown on the surface is found to be $\sim 1.7 \mathrm{~nm}$. Here, we note that CNT horizontally grown on the surface was fabricated based on the optimal synthesis condition as described above.

Figure 5 depicts the results of 1-dimensional (1D) spectra and 2-dimensional (2D) mapping images from Raman spectroscopy on the samples that were prepared as schematically shown in Figure 1(b). Specifically, the Raman images were shown for a pristine CNT (Figures 5(a)-5(c)), CNT functionalized with $\mathrm{NH}_{2}$-diazonium (Figures 5(d)-5(f)), and CNT chemically modified with RITC molecules (Figures 5(g)5(i)), respectively. In Raman spectroscopy, the G-band corresponds to planar vibrations of carbon atoms and is present in most graphite-like materials. The D-band is present in all graphite-like carbons and originates from structural defects. The radial breathing mode (RBM) corresponds to radial expansion-contraction of the nanotube. And the $G^{\prime}$-band arises from a two-phonon, intervalley, second-order Raman scattering process $[25,26]$. From these images, we have the following observations: first, when the carbon nanotubes in the HACNT array were functionalized with $\mathrm{NH}_{2}$-diazonium, the overall G-band intensities decreased over the whole spectral regime investigated, compared to the bare ones (Figures $5(\mathrm{a})$ and $5(\mathrm{~d}))$. However, the number density of bright spots in the D-band images (Figures 5(b) and 5(e)) increased, which would probably correspond to the region with $\mathrm{NH}_{2}$ diazonium functionalization. These results indicate that the $\mathrm{NH}_{2}$-diazonium functionalization would create the defect sites on the surface of carbon nanotubes. Overall, both the G-band and D-band images (Figures 5(g) and 5(h)) of the carbon nanotubes functionalized with $\mathrm{NH}_{2}$-diazonium and a subsequent RITC molecules show lower spectral intensities compared to those mentioned above. A possible reason for this would be a large amount of loss of the carbon 


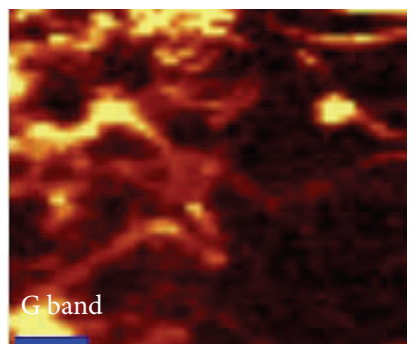

(a)



(d)

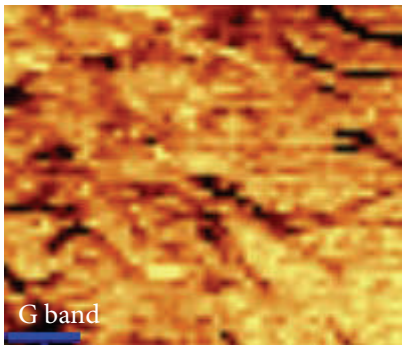

(g)

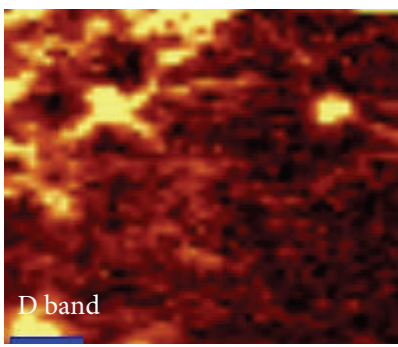

(b)



(e)

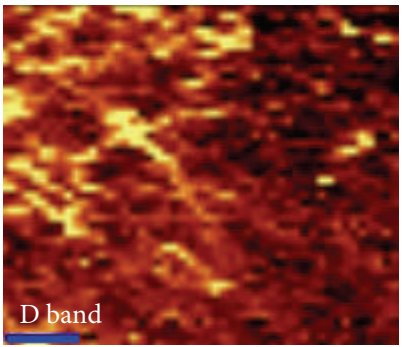

(h)

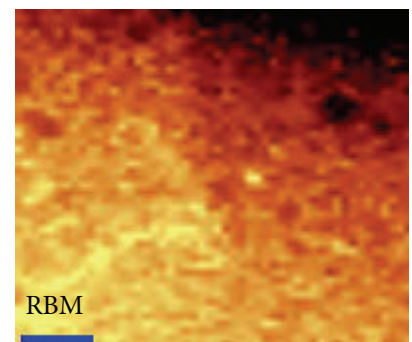

(c)

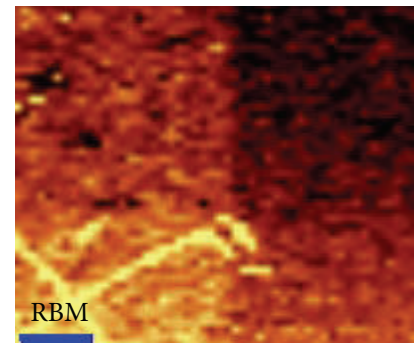

(f)

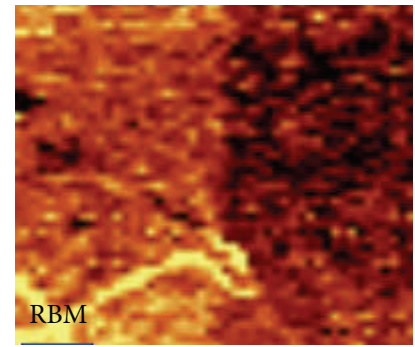

(i)

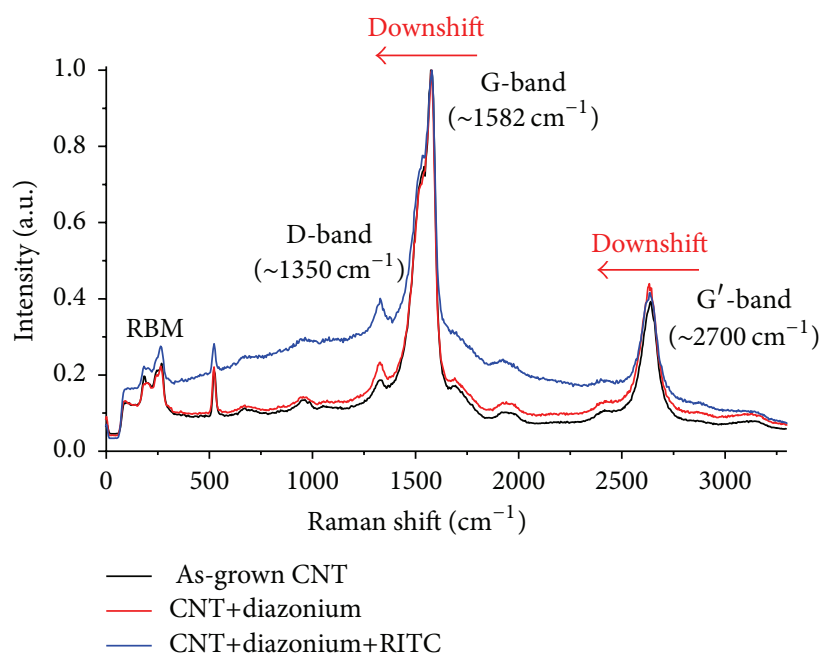

(j)

FIGURE 5: 2D Raman mapping images recorded at ((a), (b), (c)) intact individual carbon nanotubes in the HACNT array, ((d), (e), (f)) the same tubes functionalized with $\mathrm{NH}_{2}$-diazonium, and $\left((\mathrm{g}),(\mathrm{h})\right.$, (i)) the same tubes functionalized with $\mathrm{NH}_{2}$-diazonium/rhodamine dye in sequence. All scale bars represent $2 \mu \mathrm{m}$. (j) Three Raman spectra for each sample are depicted, indicating radial breathing mode (RBM), D-band, G-band, and $\mathrm{G}^{\prime}$-band. 
nanotubes during the RITC functionalization. But it is still observed that the D-band intensities in some of the regions (Figure 5(h)) became brighter than in those shown in Figure 5(e), revealing the higher degree of the functionalization. The $\mathrm{NH}_{2}$-diazonium and a subsequent functionalization of RITC molecules on the carbon nanotubes seem to produce the RBM images with little difference. Figure 5(j) depicts the representative three 1D Raman spectra of each sample discussed above. Interestingly, it was observed that the D/G-band intensity ratio increased, and the $\mathrm{G}^{\prime}$-band position downshifted $\left(\sim 15-20 \mathrm{~cm}^{-1}\right)$ with both $\mathrm{NH}_{2}$-diazonium and RITC functionalization (the resolution of the system was $1 \mathrm{~cm}^{-1}$ ). It should be noted here that the functionalization of CNT surface with $\mathrm{NH}_{2}$-diazonium would probably act as a defect site onto a SWNT surface, which makes the D-/G-band intensity ratio increase in the Raman spectra. Furthermore, the reason for both G-band and $\mathrm{G}^{\prime}$-band can be attributed to the fact that $\mathrm{NH}_{2}$-diazonium functionalization would donate electrons into carbon atom (n-doped) in a SWNT; therefore the carbon-carbon (C-C) bond at the edge of a SWNT would become extended which would directly cause the Raman softening (i.e., Raman downshift) [20,27].

\section{Conclusion}

In this work, we have determined optimal synthetic conditions of the CNT horizontally aligned on the surface with quantity control of the mixed gases of $\mathrm{H}_{2}$ and $\mathrm{CH}_{4}$ injected. It was revealed that the formation of amorphous-like carbon was critically affected by gas condition such as the flow rate of injected gases and ratios of gas mixture. It is expected that the optimal synthetic conditions studied in this work enable us to prepare the high-quality CNT-patterned surface, which may be employed for selective and sensitive biosensing applications. In addition, it should be noted that the HACNT array platform developed in this work would offer another potential in developing nanoscale light source, where light emission results from electron-hole carrier recombination. This property should enable electroluminescence (EL) in the array, which can detect subtle changes from the environments (i.e., biological and chemical). This implies that CNTpatterned surface described in this work can be considered for establishing a nanoscale optical device. Our study may provide insight into optimization of the synthesis of the HANCT array, which enables not only highly sensitive detection platforms for sensing diseases-related biomolecules, but also a new type of nanoscale light source that are able to detect environmental changes. Future work will be directed towards further development of HACNT-based biosensing platform, which may allow for diagnosis of fatal diseases such as cancer.

\section{Competing Interests}

The authors declare that they have no competing interests.

\section{Authors' Contributions}

Dawoon Jung and Hyun Woo Park contributed equally to this work.

\section{Acknowledgments}

Jae-Hee Han appreciates the financial support from CivilMilitary Technology Cooperation Program (Grant no. 14CM-EB-08), Agency for Defense Development through Chemical and Biological Defense Research Center, and National Research Foundation of Korea (NRF) under Grant no. 2012R1A1A1015734. Chang Young Lee acknowledges the financial support from NRF under Grant no. 2013R1A1A2073264. Taeyun Kwon appreciates the financial support from the National Research Foundation of Korea (NRF) under Grant no. 2015R1A2A1A15052758.

\section{References}

[1] K. Eom, H. S. Park, D. S. Yoon, and T. Kwon, "Nanomechanical resonators and their applications in biological/chemical detection: nanomechanics principles," Physics Reports, vol. 503, no. 4-5, pp. 115-163, 2011.

[2] J.-S. Park, M. K. Cho, E. J. Lee et al., "A highly sensitive and selective diagnostic assay based on virus nanoparticles," Nature Nanotechnology, vol. 4, pp. 259-264, 2009.

[3] S. Wang, H. Wang, J. Jiao et al., "Three-dimensional nanostructured substrates toward efficient capture of circulating tumor cells," Angewandte Chemie-International Edition, vol. 48, no. 47, pp. 8970-8973, 2009.

[4] G. Lee, K. Eom, J. Park et al., "Real-time quantitative monitoring of specific peptide cleavage by a proteinase for cancer diagnosis," Angewandte Chemie International Edition, vol. 51, no. 24, pp. 5837-5841, 2012.

[5] S. P. Fodor, R. P. Rava, X. C. Huang, A. C. Pease, C. P. Holmes, and C. L. Adams, "Multiplexed biochemical assays with biological chips," Nature, vol. 364, no. 6437, pp. 555-556, 1993.

[6] M. Schena, D. Shalon, R. W. Davis, and P. O. Brown, "Quantitative monitoring of gene expression patterns with a complementary DNA microarray," Science, vol. 270, no. 5235, pp. 467-470, 1995.

[7] D. Liu, A. Bruckbauer, C. Abell et al., "A reversible pH-driven DNA nanoswitch array," Journal of the American Chemical Society, vol. 128, no. 6, pp. 2067-2071, 2006.

[8] D. Zhou, K. Sinniah, C. Abell, and T. Rayment, "Label-free detection of DNA hybridization at the nanoscale: a highly sensitive and selective approach using atomic-force microscopy," Angewandte Chemie - International Edition, vol. 42, no. 40, pp. 4934-4937, 2003.

[9] S. Husale, H. H. J. Persson, and O. Sahin, "DNA nanomechanics allows direct digital detection of complementary DNA and microRNA targets," Nature, vol. 462, no. 7276, pp. 1075-1078, 2009.

[10] A. K. Sinensky and A. M. Belcher, "Label-free and high-resolution protein/DNA nanoarray analysis using Kelvin probe force microscopy," Nature Nanotechnology, vol. 2, no. 10, pp. 653-659, 2007.

[11] C. Leung, H. Kinns, B. W. Hoogenboom, S. Howorka, and P. Mesquida, "Imaging surface charges of individual biomolecules," Nano Letters, vol. 9, no. 7, pp. 2769-2773, 2009.

[12] O. K. Wong, M. Guthold, D. A. Erie, and J. Gelles, "Interconvertible lac repressor-DNA loops revealed by single-molecule experiments," PLoS Biology, vol. 6, no. 9, article e232, 2008. 
[13] J. Park, J. Yang, G. Lee et al., "Single-molecule recognition of biomolecular interaction via kelvin probe force microscopy," ACS Nano, vol. 5, no. 9, pp. 6981-6990, 2011.

[14] K. Nam, K. Eom, J. Yang et al., "Aptamer-functionalized nanopattern based on carbon nanotube for sensitive, selective protein detection," Journal of Materials Chemistry, vol. 22, no. 44, pp. 23348-23356, 2012.

[15] T. Kwon, J. Park, G. Lee et al., "Carbon nanotube-patterned surface-based recognition of carcinoembryonic antigens in tumor cells for cancer diagnosis," The Journal of Physical Chemistry Letters, vol. 4, no. 7, pp. 1126-1130, 2013.

[16] P. Avouris, M. Freitag, and V. Perebeinos, "Carbon-nanotube photonics and optoelectronics," Nature Photonics, vol. 2, no. 6, pp. 341-350, 2008.

[17] X. Xie, A. E. Islam, M. A. Wahab et al., "Electroluminescence in aligned arrays of single-wall carbon nanotubes with asymmetric contacts," ACS Nano, vol. 6, no. 9, pp. 7981-7988, 2012.

[18] K. Eom, K. Nam, H. Jung et al., "Controllable viscoelastic behavior of vertically aligned carbon nanotube arrays," Carbon, vol. 65, pp. 305-314, 2013.

[19] W. J. Hong, Y. X. Xu, G. W. Lu, C. Li, and G. Q. Shi, “Transparent graphene/PEDOT-PSS composite films as counter electrodes of dye-sensitized solar cells," Electrochemistry Communications, vol. 10, no. 10, pp. 1555-1558, 2008.

[20] B. Das, R. Voggu, C. S. Rout, and C. N. R. Rao, "Changes in the electronic structure and properties of graphene induced by molecular charge-transfer," Chemical Communications, no. 41, pp. 5155-5157, 2008.

[21] Y.-J. Do, J.-H. Lee, H. Choi et al., "Manipulating electron transfer between single-walled carbon nanotubes and diazonium salts for high purity separation by electronic type," Chemistry of Materials, vol. 24, no. 21, pp. 4146-4151, 2012.

[22] M. F. Al-Kadhemy, I. F. Alsharuee, and A. A. D. Al-Zuky, "Analysis of the effect of the concentration of rhodamine B in ethanol on the fluorescence spectrum using the 'Gauss Mod' function," Journal of Physical Science, vol. 22, no. 2, pp. 77-86, 2011.

[23] B. C. Liu, S. C. Lyua, T. J. Lee et al., "Synthesis of single- and double-walled carbon nanotubes by catalytic decomposition of methane," Chemical Physics Letters, vol. 373, no. 5-6, pp. 475479, 2003.

[24] J.-H. Han, C. H. Lee, D. Jung et al., "NH3 effect on the growth of carbon nanotubes on glass substrate in plasma enhanced chemical vapor deposition," Thin Solid Films, vol. 409, no. 1, pp. 120-125, 2002.

[25] P. C. Eklund, J. M. Holden, and R. A. Jishi, "Vibrational modes of carbon nanotubes; spectroscopy and theory," Carbon, vol. 33, no. 7, pp. 959-972, 1995.

[26] M. S. Dresselhaus, G. Dresselhaus, R. Saito, and A. Jorio, "Raman spectroscopy of carbon nanotubes," Physics Reports, vol. 409, no. 2, pp. 47-99, 2005.

[27] A. C. Ferrari, "Raman spectroscopy of graphene and graphite: disorder, electron-phonon coupling, doping and nonadiabatic effects," Solid State Communications, vol. 143, no. 1-2, pp. 47-57, 2007. 

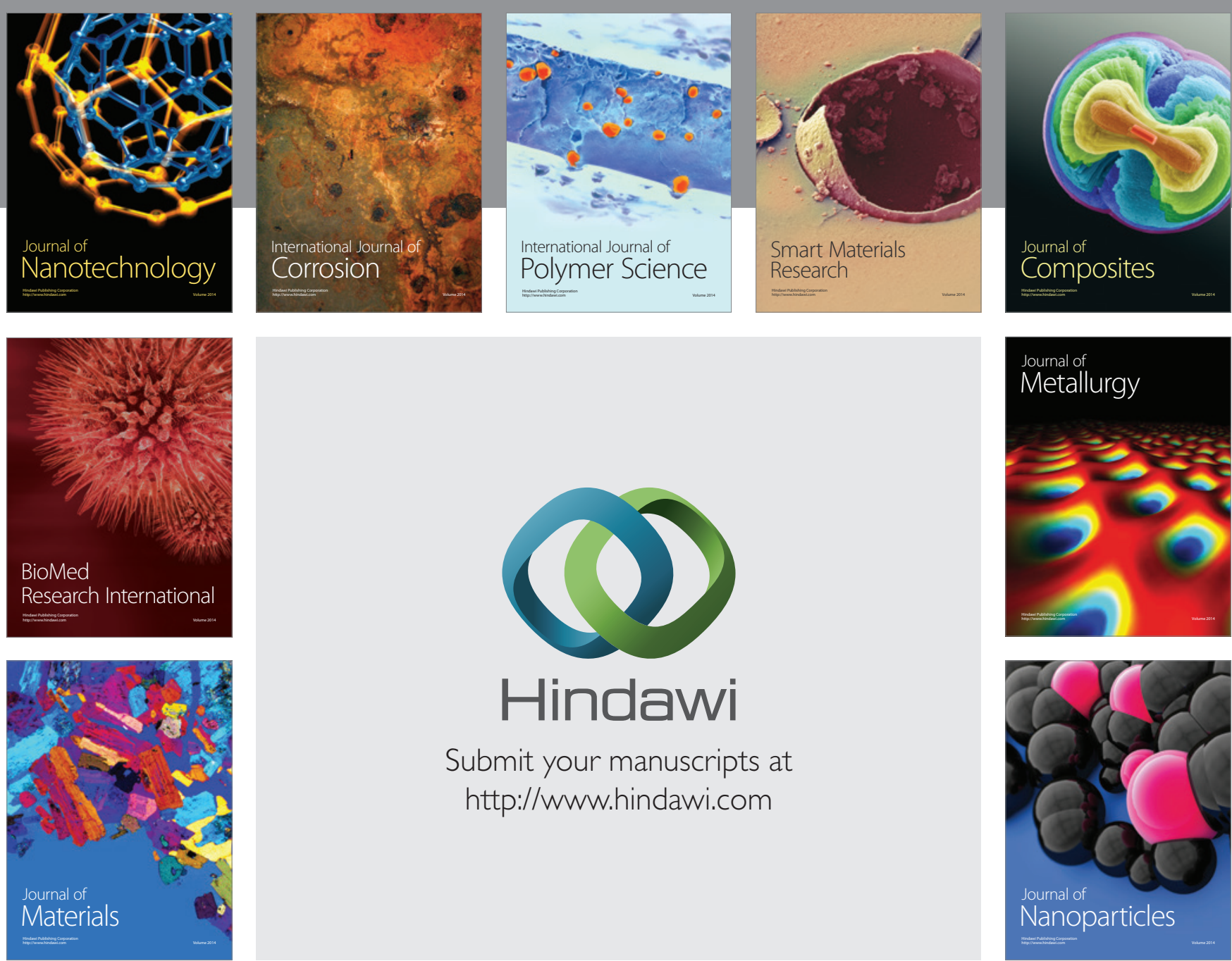

\section{Hindawi}

Submit your manuscripts at

http://www.hindawi.com

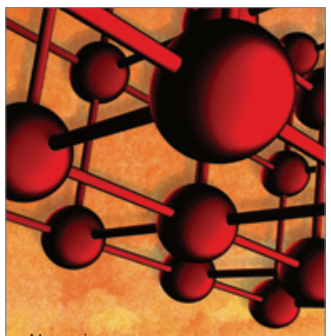

Materials Science and Engineering
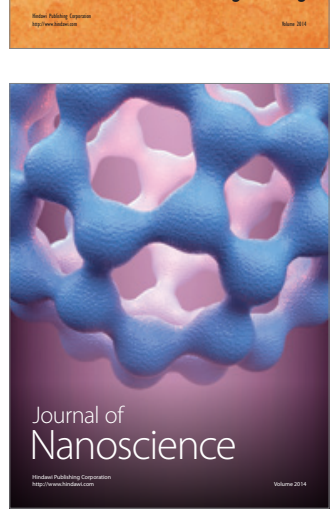
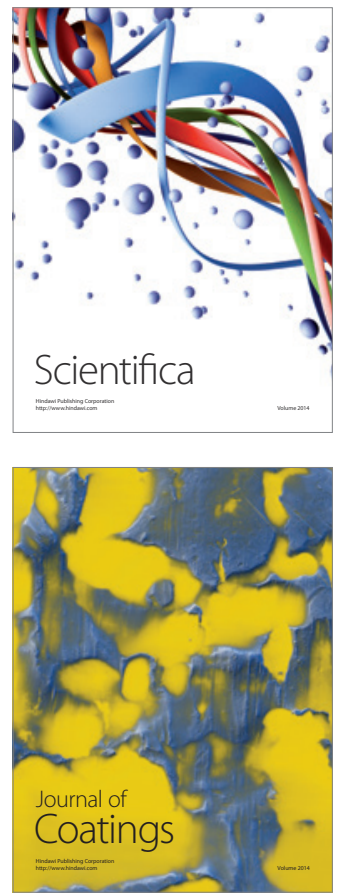
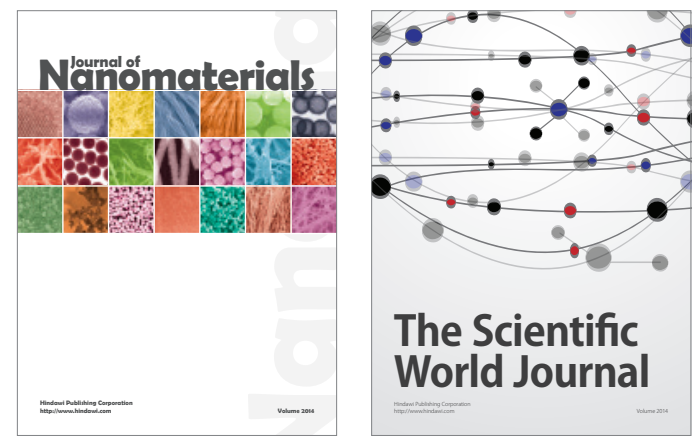

The Scientific World Journal
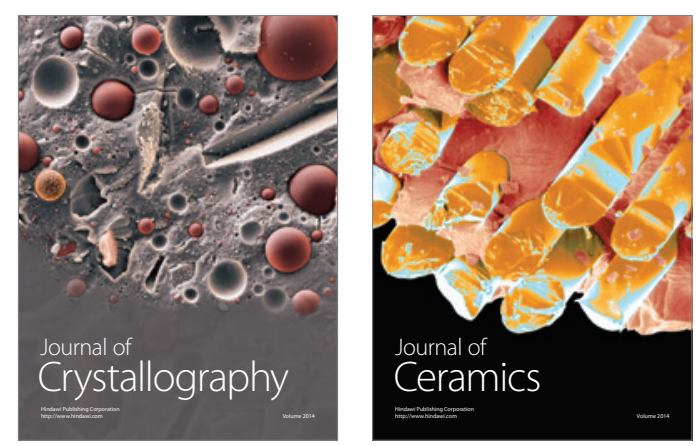
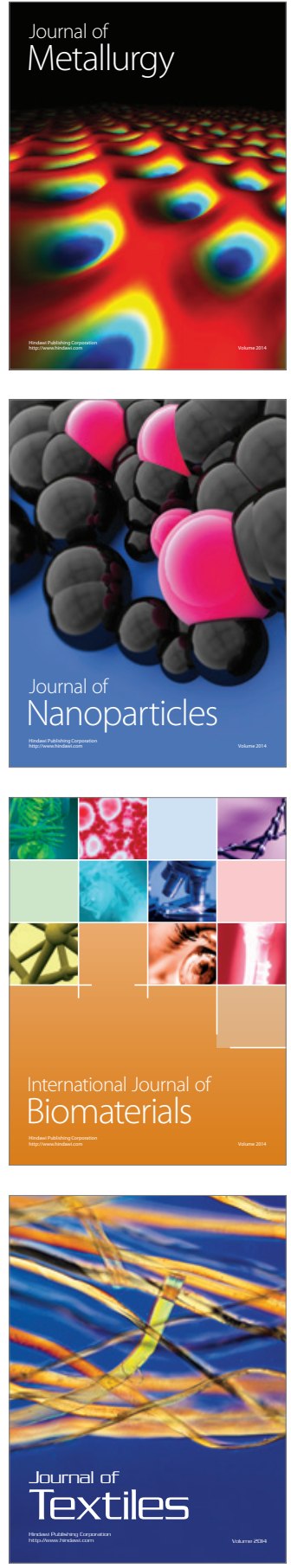